\title{
A Proposed Model to eliminate the Confusion of Hematological Diseases in Thin Blood Smear by Using Deep Learning-Pretrained Model
}

Mahir M. Sharif ${ }^{1}$ *, Hassan Abdelrhman Mohammed ${ }^{2}$ and Eltahir Mohmmed Hussein ${ }^{3}$

${ }^{1}$ Dept. of Computer Sciences, Faculty of Computer Sci. \& Information Tech, OIU University, Omdurman, Sudan

${ }^{2}$ Dept. of Computer Sciences, Faculty of Computer Sci. \& Information Tech., Karary University, Omdurman, Sudan

${ }^{3}$ Dept. of Electrical and Electronic Engineering, Faculty of Engineering, Sudan SUST University, Khartoum, Sudan

Email address:

mahiralsharif@oiu.edu.sd (Mahir M. Sharif)

*Corresponding author

To cite this article:

Mahir M. Sharif, Hassan Abdelrhman Mohammed and Eltahir Mohmmed Hussein. A Proposed Model to eliminate the Confusion of Hematological Diseases in Thin Blood Smear by Using Deep Learning-Pretrained Model, Omdurman Islamic University Journal, Vol. 18, No. 1, 2022, pp. 81-92 doi:10.52981/oiuj.v18i1.2038

Received: 10 09, 2021; Accepted: 11 12, 2021; Published: 02 16, 2022.

\begin{abstract}
This research aimed to developing and designing a model for resolving the confusion between hematology in a thin blood smear by means of a pre-defined deep learning model for detection and identification of hematological diseases in thin blood smear images for accurate diagnosis of the different diseases that leukemia and malaria were performed as a sample. There are catastrophic consequences that may lead to death as a result of a mistake in diagnosis and confusion in the knowledge of the disease in particular, especially in hematology, where another disease that was not originally found in the sample is identified for the similarity, which results in surgery and sometimes the administration of drugs in error. In this work, an image processing system was developed to identify patients with malaria and leukemia. The techniques in deep learning have been implemented where the CNN (Alexnet and Resnet50) image recognition model was applied to detect patterns and extract features of the different types of malaria and leukemia from the images. And that is through developing algorithms to distinguish between the two diseases, discovering the presence of similarities in the patterns of stages and the different types of malaria and leukemia in blood images, and reaching to solve the problem of confusion by training, verification, and testing using the mutual verification system that uses three folds. The system achieved an accuracy of $94.3 \%$ for Resnet50 and 92.3 for Alexnet in detecting and classifying the types and stages of the two diseases (malaria and leukemia). And 100\% to distinguish between them.
\end{abstract}

Keywords: Deep Learning, CNN, Leukemia, Malaria, Thin Blood Smear Recognition 


\section{Introduction}

The clinical investigation of hematological diseases is fundamentally based on laboratory blood analyses and also the most skilled hematology professional can face patterns, deviations, and relationships between the increasing numbers of blood parameters that current laboratories measure. In contradiction, machine learning algorithms can easily handle centuries of attributes (parameters), and they are able of detecting and using the interactions among these various attributes, which makes this field of medicine very interesting for machine learning applications. The researchers hypothesized that the "fingerprint" of a unique hematological disease found in the values of blood test results would be sufficient for a machine learning-based predictive model to propose a plausible diagnosis, presented that the machine was trained on an adequately large dataset of medical problems that include clinical laboratory blood analyses and that were labeled with an accurate diagnosis defined by a hematology specialist who has utilized all of the diagnostic methods necessary to validate it[1].The microscopic investigation of blood smears provides diagnostic information regarding patients' health status. The examination results of the differential blood count expose a wide range of critical hematic pathologies. For example, the carriage of infections, leukemia, and some particular kinds of cancers can be diagnosed based on the analysis outcomes and the count of white blood cells. The expert operatives do the traditional method for the differential blood number. They use a microscope and count the rate of any cell counted within an area of concern in smears. This hand-operated counting process is very tedious and slow. Also, cell classification and counting efficiency may depend on the skills and experiences of the operators. Therefore, the necessity of an automatic differential counting method becomes inevitable[2]. Leukemia is a kind of cancer associated with white blood cells that begin in the bone essence and affects both children and adults. Leukemia can be classified into dangerous or chronic categories based on how quickly it progresses. There are four varieties of leukemia, namely, Acute My elogenous Leukemia (AML), Acute Lymphoblastic Leukemia (ALL),Chronic Myeloid Leukemia (CML) and Chronic Lymphocytic Leukemia (CLL)[3, 4].There are two main kinds of lymphoma: Hodgkin lymphoma and non-Hodgkin lymphoma[5].

Malaria is a hemoglobin disease affected by the Plasmodium parasites transferred through the bite of the female Anopheles mosquito. Microscopists commonly examine thick and thin blood smears to diagnose illness and compute parasitemia. Nevertheless, their accuracy depends on smear quality and expertise in classifying and counting parasitized and uninfected cells. Such an examination could be arduous for large-scale diagnoses were resulting in low quality [6]. There are Four species of Plasmodium that have largely been identified to infect humans. These are Plasmodium falciparum (P.F), Plasmodium vivax (P.V), Plasmodium ovale (P.O), and Plasmodium malariae (P.M). Nevertheless, all of them go through the similar life stages in peripheral human blood (Ring, T.zoitesS.izonts, G.cytes). Sometimes, it is hard to separate between species in the same life stages, especially the initial stage [7].

\section{Literature Review}

Many techniques for the deep learning are proposed in literature. Others in [8]proposes to develop a deep learning model to discuss the blood cell analysis problem, which is one of the several challenging problems in blood investigation. A CNN-based structure is created to automatically classify the blood cell images into subtypes of the cells. Experiments are transferred on a dataset of 13k images of blood cells by their subtypes, and the results show that our suggested model produce better results in times of evaluation parameters.

Study in [9] introduces three consecutive pre-processing considerations namely are color distortion, bounding box distortion (crop), and image flipping following. In the second phase, white blood cell 
recognition was achieved with hierarchy topological feature extraction using Origin and ResNet structures. Finally, the results obtained from the initial analysis of cell classification with (11200) training samples and 1244 white blood cells evaluation dataset are presented in confusion matrices and performed using accuracy rate. False-positive with the classification frame validated with experiments carried on low-quality blood images sized $320 \times 240$ pixels. The challenging cell detection task's deferential outcomes indicate a significant achievement in using Beginning and ResNet architecture with proposed settings. Our framework detects on average $100 \%$ of the four main white blood cell types using ResNet V1 50 while also encouraging alternative result with $99.84 \%$ and $99.46 \%$ accuracy rate obtained with ResNet V1 152 and ResNet 101, individually with 3000 epochs and fine-tuning all layers. Further mathematical confusion matrix tests showed that this work achieved 1, 0.9979, 0.9989 sensitivity values when area under the curve (AUC) rates over 1, 0.9992, 0.9833 on three proposed systems. The modern work also shows negligible and small false negative $0,2,1$, and plentiful false positive with $0,0,5$ values in Leukocytes detection.

The work in [10] develop the computational method based on deep convolution neural networks for breast cancer histology image classification. Hematoxylin and eosin-stained breast histology microscopy image dataset are provided as a part of the ICIAR Grand Challenge on Breast Cancer Histology Images. Our system utilizes many deep neural network architectures, and grade boosted trees classifier for class analysis task, at the high-sensitivity turning point. To our experience, this approach outperforms other standard methods in automated histopathological image classification.

the study in [11] used 7000 images of exception, Inception-V3, ResNet-50,NasNetMobile, VGG-16, and AlexNet models for verifying and analysis. These are standard models that classify the image accuracy and use a rotational method to enhance the execution of validation and the training dataset with convolutional neural network models. exception, using the state of the creation activation function (Mish) and optimizer (Nadam), profited thee. Activeness, as found by the results of the convolutional neural model evaluation of these models for classifying the malaria infection from thin blood smear images. In phases of the performance, recall, accuracy, sureness, and F1 measure, a composite score of $99.28 \%$ was obtained. Consequently, $10 \%$ of all non-dataset training and testing images were evaluated utilizing this pattern. Essential aspects for editing a computer-aided diagnostic to produce an excellent malaria detection method have been found, confirmed by a $98.86 \%$ accuracy level.

The work in [12] presents a novel three-stage pipeline to (1) section erythrocytes, (2) harvest and cover them, and (3) characterize them into malaria-infected or not. The first and third steps included the plan, preparing, approval, and testing of a Segmentation Neural Network and a Convolutional Neural Network without any preparation utilizing a Graphics Processing Unit. Division accomplished a worldwide exactness of $93.72 \%$ over the test set and the particularity for jungle fever identification in red platelets (RBCs) was $87.04 \%$. This work shows the potential that profound learning has in the advanced pathology field and opens the route for future upgrades, just as for expanding the utilization of the made organizations.

Authors [13]proposes two mechanized grouping models dependent on blood infinitesimal pictures to identify leukemia by utilizing move learning, instead of customary methodologies that have a few impediments. In the primary model, blood tiny pictures are pre-handled; at that point, highlights are separated by a pre-prepared profound convolutional neural organization named AlexNet, which makes groupings as indicated by various notable classifiers. In the subsequent model, after pre-handling the pictures, AlexNet is tweaked for both element extraction and characterization. Trials were directed on a 
dataset comprising of 2820 pictures affirming that the subsequent model performs better compared to the first due to $100 \%$ arrangement precision.

The study in [14] proposed a novel methodology dependent on regular computerized picture handling strategies and AI calculations to naturally distinguish intense lymphoblastic leukemia from fringe blood smear pictures. To beat the best difficulties in the division stage, the researchers carried out broad pre-preparing and acquainted a three phase filtration calculation with accomplish the best division results. Besides, sixteen vigorous highlights were extricated from the pictures in the manner that hematological specialists do, which altogether expanded the ability of the classifiers to perceive leukemic cells in infinitesimal pictures. To play out the order, the researchers applied two customary AI classifiers, the counterfeit neural organization and the help vector machine. The two techniques arrived at an explicitness of $95.31 \%$, and the affectability of the help vector machine and fake neural organization arrived at 98.25 and $100 \%$, individually .

\section{MATERIALS AND METHOD}

System architecture used for pre-trained model for detection and classification of diseases in medical images involves the following main steps: an image input layer, CNN pre-trained model, classification, and output layer. The block diagram shown in Fig 1 illustrates all system steps. Matlab is used in all system implementation steps.

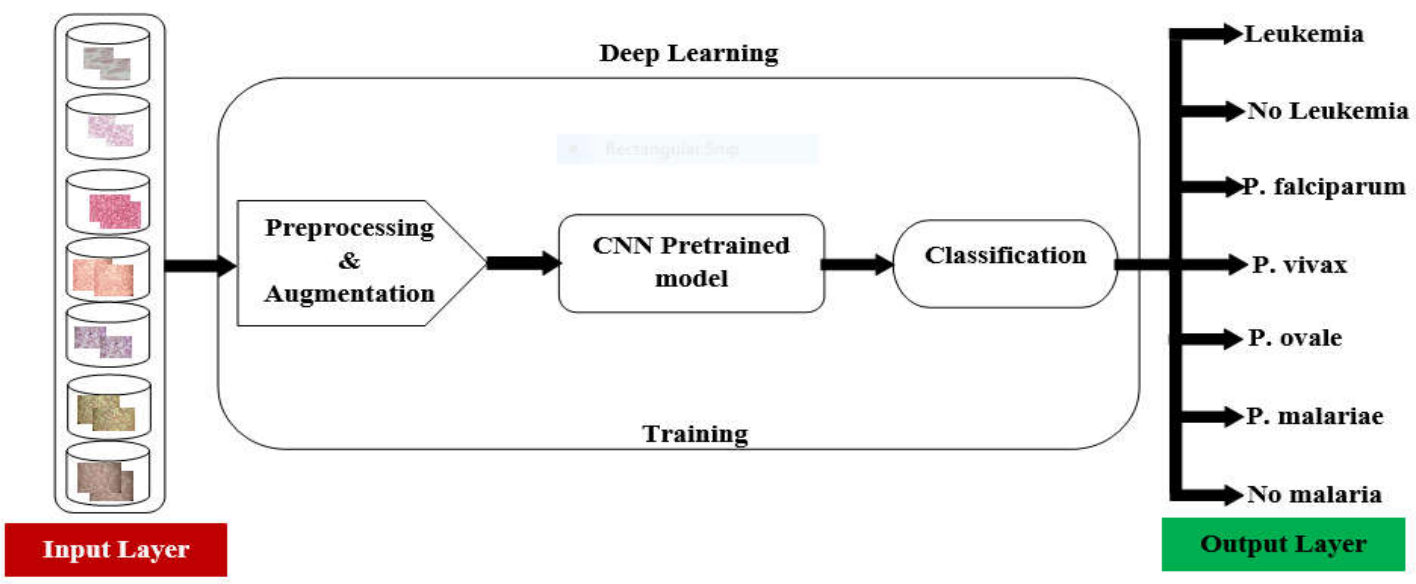

Figure 1:. Model block architecture

\section{A. Deep Learning \& CNN Pretrained Models}

Deep Learning is an appropriate machine learning subfield, in new learning description of information indicating "consecutive" layer learning of description. Deep Learning allows computational models organized in numerous readiness layers to examine report spoke to by the extraordinary degree of abstraction[15]. Deep-learning innovation for AI has pulled in incredible consideration as it continually breaks records in a collection of necessary benchmark tests. As one kind of technique that fulfills the prerequisites of deep learning, $\mathrm{CNN}$ is directly a cutting edge profound learning structure applied to a broad scope of fields, for example, programmed machine construction, $\mathrm{PC}$ vision, and speech recognition. Incredible execution in object location or grouping from images has been shown because CNN networks give a quicker, more vigorous, and more beneficial calculation than traditional neural networks. In CNN, 
image pixels could be straightforwardly utilized as an increase to the standard feed-forward neural networks. Although several pixels from even little image patches bring about an enormous number of community weight boundaries to be prepared, $\mathrm{CNN}$ models consolidate loads into many more modest bit channels that drastically replace the learning model[16]. In clinical imaging, the most ordinarily utilized deep learning procedures are convolutional neural networks (CNN) Compared features inside the information itself. By and large, $\mathrm{CNN}$ is better ready to distinguish features over the human eye[17].

Deep learning algorithms, in special convolutional neural networks, have swiftly become a methodology of opportunity for investigating medical images [12]. Deep learning techniques have been used for medical image analysis in several diseases to address such tasks as classification, object detection, and tissue segmentation. This review covers applications in diseases. Matlab CNN functions are used for implementation of the following steps: input dataset of grey and color medical images for (malaria) show in table 1 followed, feature extraction by applied convolution layer technique, max pooling layer and fully connected layer techniques to detection and classification the fetus health states.

\section{B. Image Dataset}

Kaggle and $U$ medical image dataset is the source of information required for the analysis and development of hematological diseases detection and classification model. RGB medical images information also provides a visualized unhealthiness of the several diseases that helps for early intervention before the risk happening. total of 600 instances with four sample of diseases (Leukemia and malaria) chosen attributes which are multivariate data types divided to 6 classing base on defect with Leukemia and malaria, but malaria in defect divided into four categories Plasmodium falciparum (P.F), Plasmodium vivax (P.V), Plasmodium ovale (P.O), and Plasmodium malariae (P.M) and normal are shown in Table I.

TABLE I. DISEASES INPUT RGB MEDICAL IMAGES

\begin{tabular}{|l|c|c|}
\hline \multirow{2}{*}{ Diseases } & \multicolumn{2}{c|}{ Image Size } \\
\cline { 2 - 3 } & Alexnet & Resnet50 \\
\hline Leukemia & $227 \times 227 \times 3$ & $224 \times 224 \times 3$ \\
\hline Plasmodium falciparum & $227 \times 227 \times 3$ & $224 \times 224 \times 3$ \\
\hline Plasmodium vivax & $227 \times 227 \times 3$ & $224 \times 224 \times 3$ \\
\hline Plasmodium ovale & $227 \times 227 \times 3$ & $224 \times 224 \times 3$ \\
\hline Plasmodium malariae & $227 \times 227 \times 3$ & $224 \times 224 \times 3$ \\
\hline Normal & $227 \times 227 \times 3$ & $224 \times 224 \times 3$ \\
\hline
\end{tabular}

\section{Pre-processing}

Used in this study a mini-batch size of 512 with 8 GPUs for training our models. The initial learning rate is set to 0.01 . the researchers train with a total number of 8 epochs, and the learning rate is multiplied by 0.1 at three epochs. The weight decay is not used (set to 0 ) for training and applied this strategy to all proposed experiments - architectures which it chooses.

used the create a new model for medical images dataset is divided to 6 folders as datastore according to feature which appears for each type diseases see table I. all medical image obtained was resized to $224 \times$ 224 to apply in resnet50 and resized to $227 \times 227$ to apply in elexnet. Of the total 600 images selected from 
the dataset used in this study, cross-validation is set to 3 as the value of folds, which means two-third were used for training, while the other One third was used for validation. Hence, 400 images were used to train the network, and 200 images were used for validation.

\section{EXPERIMENTS}

The experiment was conducted using Matlab version R2018b program in all stages of the investigation from preparation to reaching the results, which can be described as follows:

\section{A. labeled and adjust number of images in Datastore}

Start the proposed Experiment by Create an Image Datastore to help the manage data. Because Image Datastore operates on image file locations, images are not loaded into memory until reading, making it efficient for extensive image collections. This step is significant, so I proposed a labeled base on diseases in table I divided into 6 categories. Each one contends 100 medical images for different disease and normal, so contains the images and the category labels associated with each image. The labels are automatically assigned from the folder names of the image files. And summarize the number of images per category. present in table II.

\section{TABLE II. CATEGRY LABELS ACCORDING TO TYPE OF DISEASES}

\begin{tabular}{|c|c|}
\hline Label & Count \\
\hline Leukemia & 100 \\
\hline Normal & 100 \\
\hline P_Falciparum & 100 \\
\hline P_Malariae & 100 \\
\hline P_Ovale & 100 \\
\hline P_Vivax & 100 \\
\hline
\end{tabular}

In the situation, obtained new medical images from the proposed system's image data Datastore an unequal number of images for each category. Let's first adjust it so that the number of images in the training set is balanced using the MATLAB function. And select an image for each file labeled to make sure. Fig 2 Below a presented example of images from 6 of the categories included in the dataset.
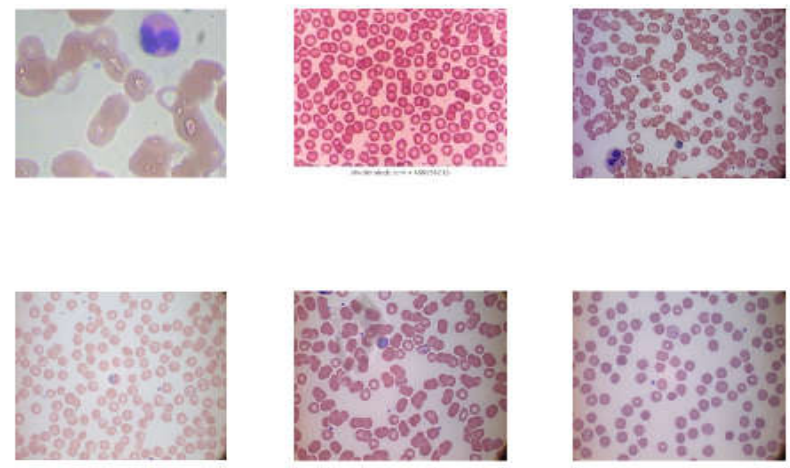

Figure 2: Selected image from each file labeled of midst ready for training 


\section{B. Training for Detection and Classification Diseases:}

Convolutional neural networks automatically learn the best deputy features from the fresh data to extract feature in thin blood smear. This study used the Alexnet and ResNet50 CNN for the proposed system method due to its state-of-the-art performance in diseases (Leukemia and \& malaria) detection in the challenging, complex detection and classification of the diseases total and individual. The researchers modified the input layer to fit the size of the extracted image objects. Our pre-training on the medical image patch dataset involved finding the set of $\mathrm{CNN}$ layer weights and used a cross-validation model with three folds.

\section{Data Augmentation}

Due to the move in the position of the diseases inside the medical images. This proposed system was used an image data augmenter that configures a set of preprocessing options for image augmentation, such as resizing, rotation, and reflection. The image Data Augmenter is used by an augmented Image Datastore functions to generate batches of augmented images. The researchers expand the number of frames using data augmentation for the images labeled in Section A Types of augmentation are: moving to the left / right direction, enlarging/reducing. Rotation and color tone correction etc., are not carried out. Horizontal movement, enlargement/reduction are performed randomly so that the number of data is increased about five times as many as the original frame number.

\section{Validation and Testing}

In this study that there are about 600 images available in this dataset. Split the dataset into 3-folds for analysis, i.e., three different algorithms of pre trainrd model would be trained using different sets of images from the dataset. This type of validation study would provide us a better estimate of our performance compared to the typical hold-out validation method.

The researchers adopt Alexnet and ResNet-50 architecture in this blog as it has proven to be highly effective for various medical imaging applications. One idea of proposed system Replacing the number of classes with 6 according to its categories and Replacing the last layers with new layers. Implemented experiment for each pre_trained model together and detailed.

\section{E. Classification of Selection diseases and normal:}

After training $\mathrm{CNN}$ Alexnet and resnet50 of proposed system for extract features in thin blood smear. The system classifies the entered images into 6 categories presented in table II so that if it has achieved an accuracy of more than $90 \%$.

\section{RESULTS AND DISCUSSION}

After implementing all the steps described in the previous section, the system is evaluated, and the final results are discussed. Results obtained from the proposed system automatically during training, validation, and testing process to get accurate results.

In elexnet the network requires input images of size 227-by-227-by-3, but the images in the image datastores have different sizes. Use an augmented image datastore to automatically resize the training images. Specify additional augmentation operations to perform on the training images: randomly flip the training images along the vertical axis, and randomly translate them up to 30 pixels horizontally and vertically. Data augmentation helps prevent the network from overfitting and memorizing the exact details of the training images. Specify the training options. For transfer learning, keep the features from the early layers of the pretrained network (the transferred layer weights). 
To slow down learning in the transferred layers, define the initial learning rate at a low value. In the previous step, it can have increased the learning rate factors for the fully connected layer to speed up learning in the new final layers. This combination of learning rate settings results in faster learning only in the new layers and slower learning in the other layers. When carrying out learning transfer, it does not need training for as many times. An epoch is a full training cycle on the entire training data set. Specify the mini-batch size and validation data. The software validates the network every Validation Frequency iteration during training. Train the network that consists of the transferred and new layers. By default, train Network uses a GPU if one is available (requires Parallel Computing Toolbox ${ }^{\mathrm{TM}}$ and a CUDA ${ }^{\circledR}$ enabled GPU with compute capability 3.0 or higher). Otherwise, it uses a CPU. It can also specify the execution environment by using the 'Execution Environment' name-value pair argument of training Options shows in fig 3 Below.

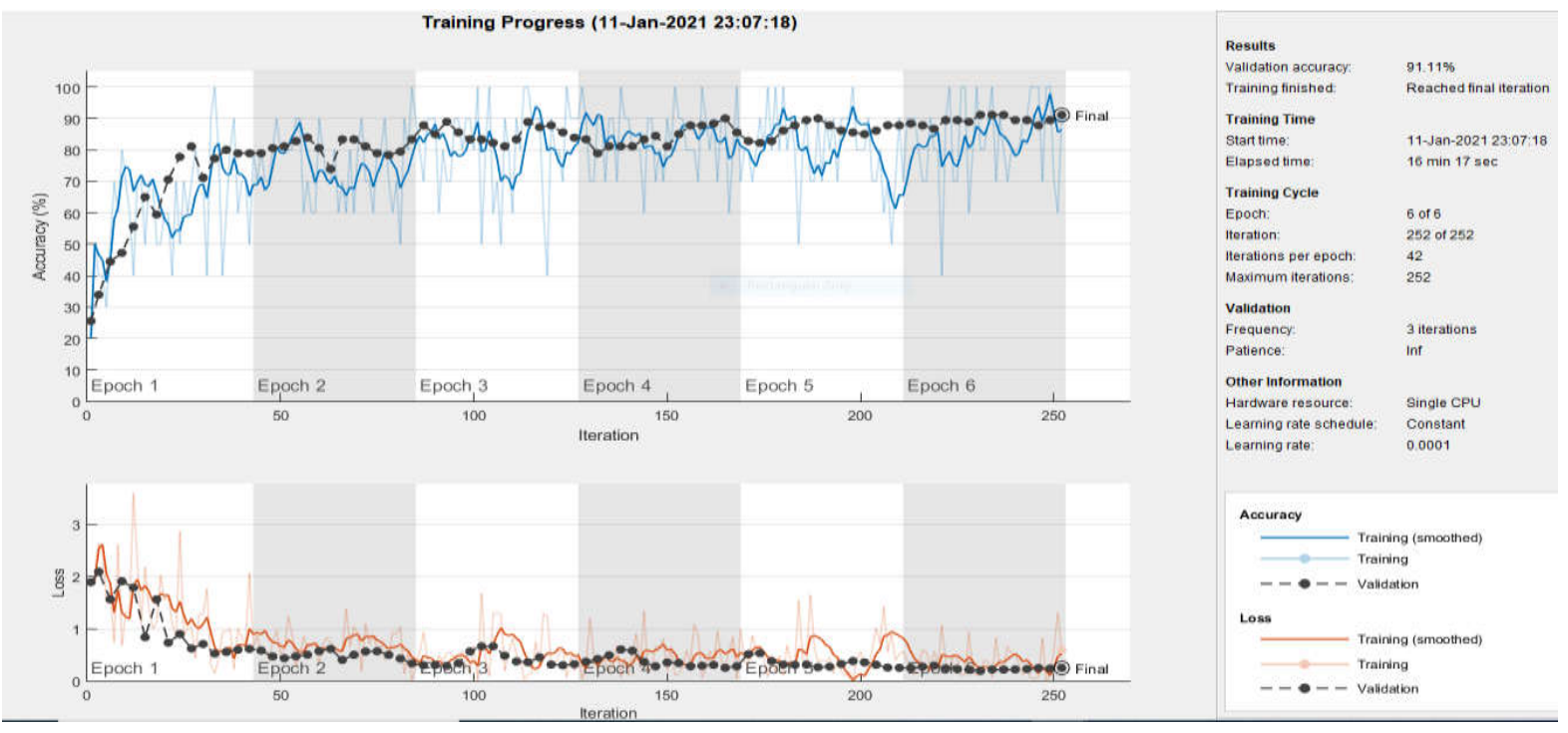

Figure 3: Alexnet Training Progress

Calculate the classification accuracy on the validation set. Accuracy is the fraction of labels that the network predicts correctly for all hematology in image datastore.

In Resnet50 used cross-validation technics by applying This is to repeat the experiment three times using the values mentioned in the previous section, as each investigation resulted in a curve for accuracy and loss. The mean was calculated for accuracy to obtain excellent accuracy due to repetition, training, and verification in each experiment. The three graphic carve are shown in Fig3, Fig4, Fig5. and The confusion matrix shown in Fig6 in grouped. and implement in details The three graphic carve are shown in Fig 7, Fig8, Fig9. and The confusion matrix shown in Fig10. The first experiment was started in split1 from proposed algorithm by selecting the first third of the test's images and the last two thirds for the training. That is the first fold for testing and the second and third fold for training. The following results are obtained through the training progress, as shown in 
Figure 4, where 32 minutes and 10 seconds were spent for five epochs and 250 repetitions, i.e., an average of 50 repetitions per epoch.
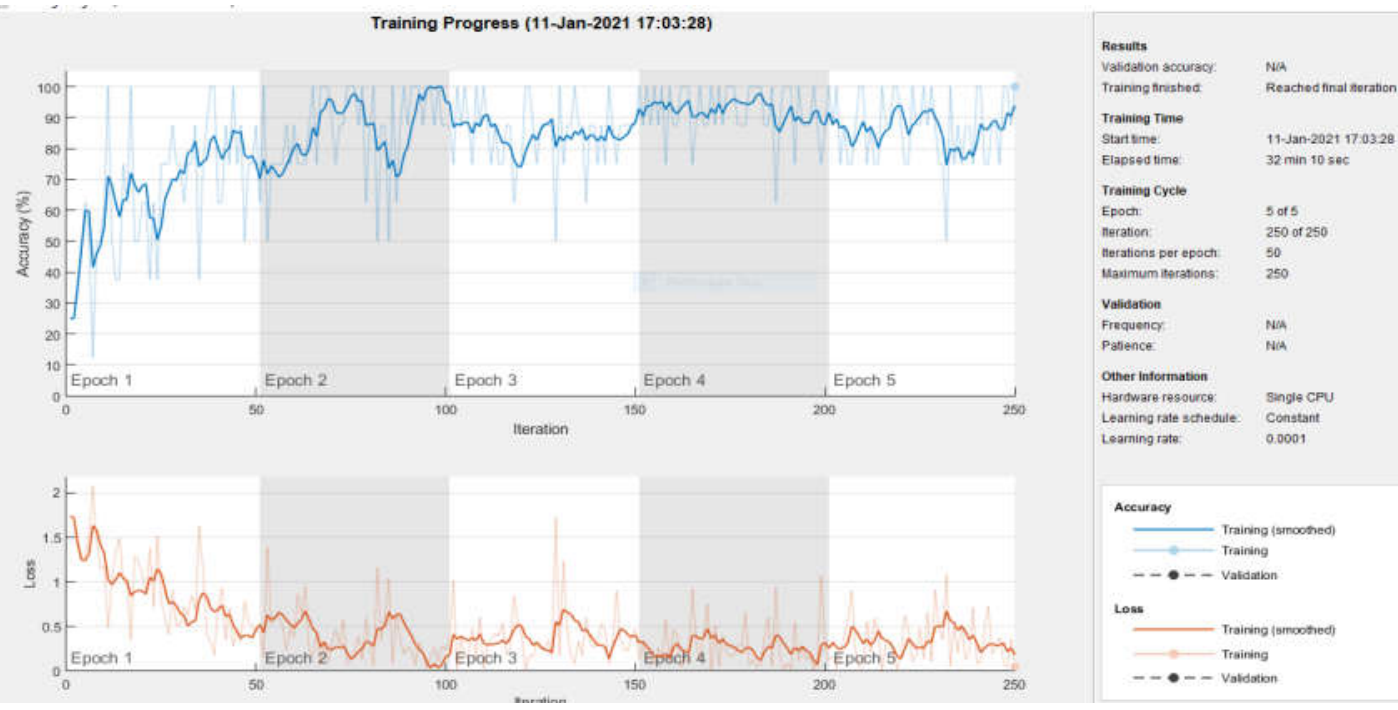

Figure 4: Training Progress in split1

The second experiment was started in split 2 from proposed algorithm by selecting the second third of the test's images and the other two thirds for the training. That is the second fold for testing and the first and third fold for training. The following results are obtained through the training progress, as shown in Figure 5, where 33 minutes and 28 seconds were spent for five epochs and 250 repetitions, i.e., an average of 50 repetitions per epoch.

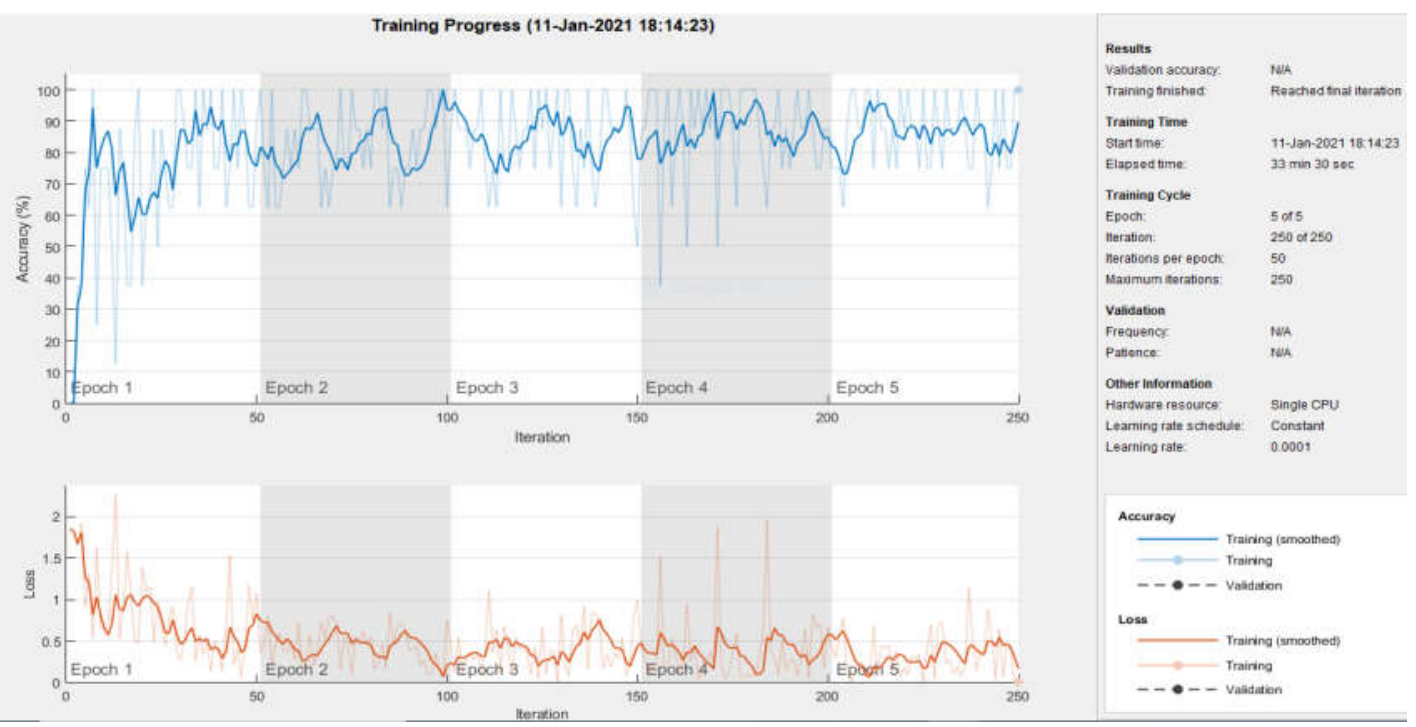

Figure 5: Training Progress in split2

The third experiment was started in split3 from proposed algorithm by selecting the third of the test's images and the other two thirds for the training. That is the third fold for testing and the first and second fold for training. The following results are obtained 
through the training progress, as shown in Figure 6, where 33 minutes and 30 seconds were spent for five epochs and 250 repetitions, i.e., an average of 50 repetitions per epoch.

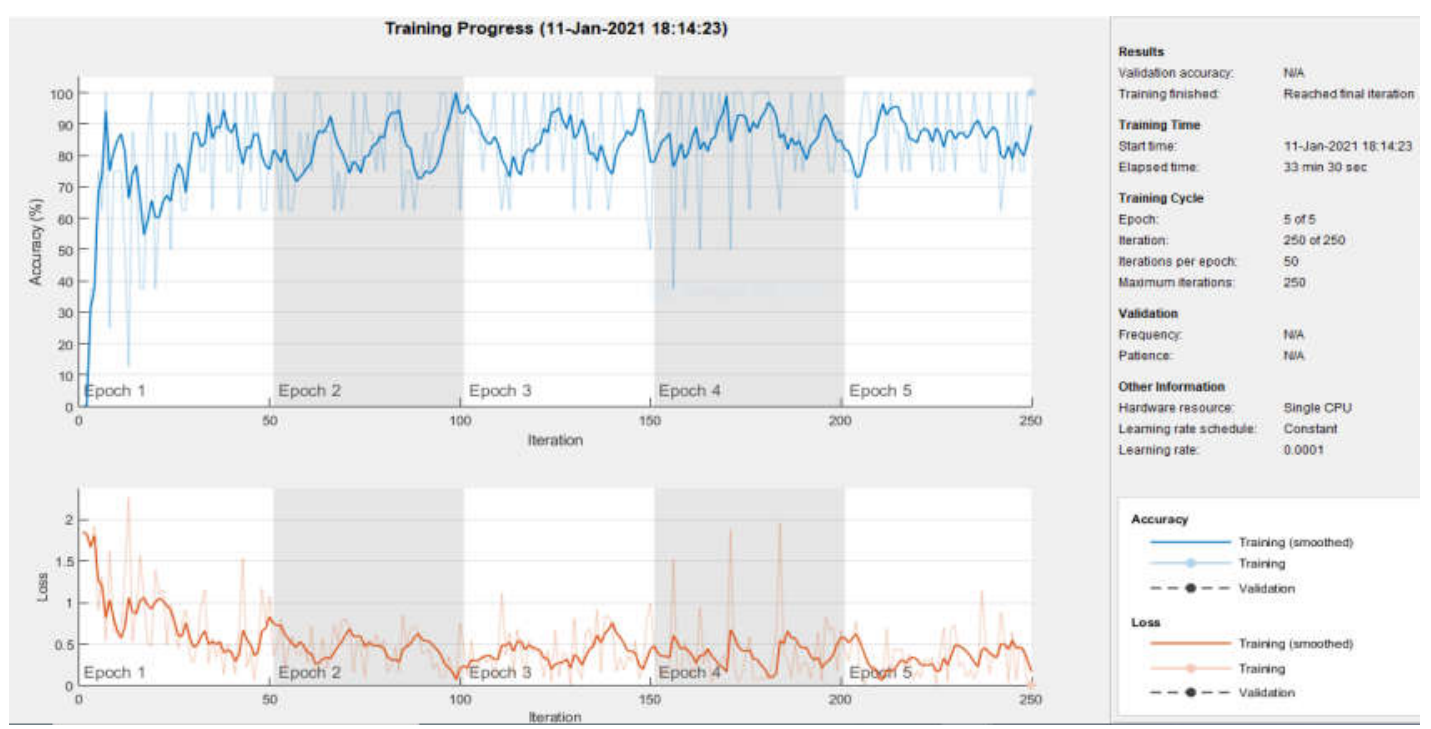

Figure 6: Training Progress in split3

\section{B. Evaluation of the Performance of the Experiments}

Measured the performance of the proposed algorithm by confusion matrix - This metric also gives a great idea of the performance in duration of accuracy and recall The researchers believe overall accuracy is an excellent pointer as the testing dataset used in this work is consistently spread (in terms of images belonging to each category of malaria and Leukemia). as shown in fig 7. Confusion matrix was achieved with an accuracy of 94.7 for 3 folds to diagnose the Leukemia and all type of malaria.

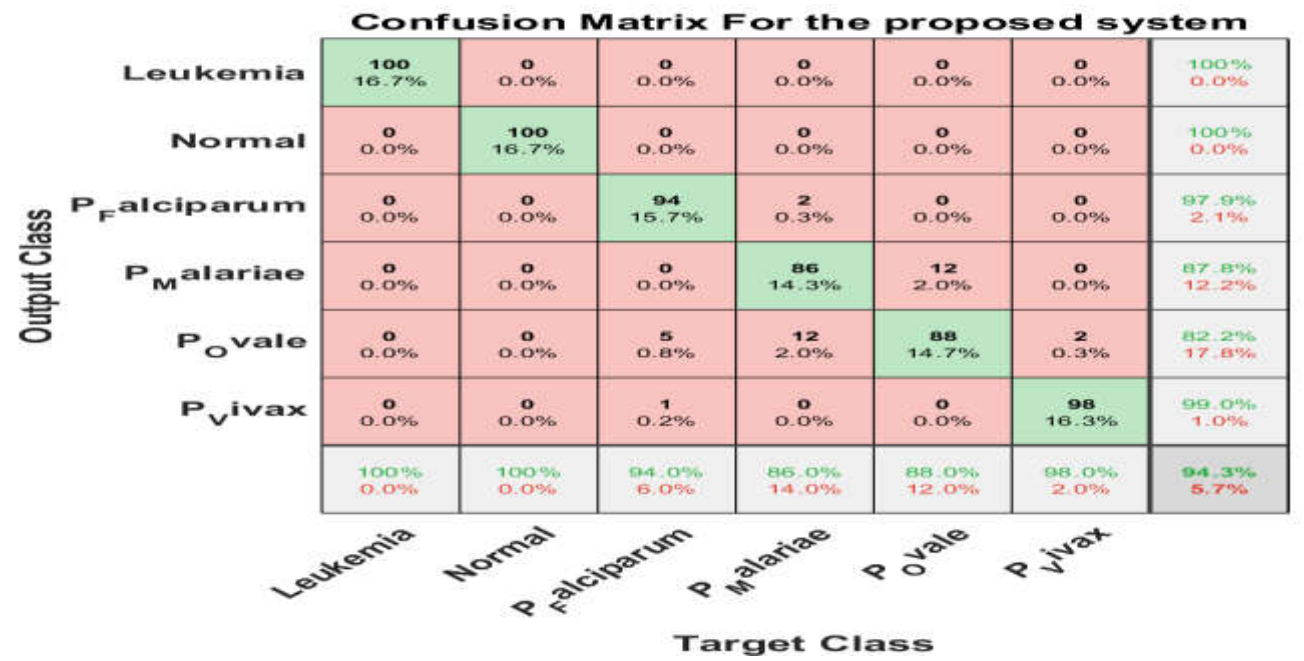

Figure 7: The confusion matrix for the proposed system 
C. Discussion

This study achieved great success in eliminating the confusion between the hematology diagnosis system which was evaluated in this work through table III and the confusion matrix presented in the evaluation section to verify its validity. This study used a new method to detect and classify Leukemia and all kinds of malaria and the readiness of the uterus into account, unlike other studies that focused on a specific part of the hematology such as the one sample or an isolated type such as the predictive of development of the hematology in future.

This study achieved great success in the different confusion between hematology in a thin blood smear. It failed in something in the several type of malaria due to the change of the enlargement of the stages organs and the similarity between the species at this stage. And solve this problem by increasing the data and using Data Augmentation after this solution achieved more than $94 \%$ accuracy for diagnosing Leukemia and all kinds of malaria acc. Similarities were found between this study and other studies in detecting the state of the hematology apparent in the use of deep learning pre trainrd model. However, see fig 7 this study was distinguished by setting the scope of the study of Leukemia and all kinds of malaria, to be exact Accurate results and proper diagnosis.

TABLE III. ACCURACY OF HEMATOLOGY (LEUKEMIA \& MALARIA)

\begin{tabular}{|c|c|c|}
\hline Diseases & Alexnet & Resnet50 \\
\hline Leukemia & $98.3 \%$ & $100 \%$ \\
\hline Normal & $100 \%$ & $100 \%$ \\
\hline P_Falciparum & $94.3 \%$ & 97.9 \\
\hline P_Malariae & $79.9 \%$ & 87.8 \\
\hline P_Ovale & $80.5 \%$ & 82.2 \\
\hline P_Vivax & $96.7 \%$ & 99.0 \\
\hline Leukemia and all & $100 \%$ & $100 \%$ \\
\hline
\end{tabular}

\section{Conclusion}

This paper presented a medical image processing system to discover and classify the state of human health in tow sample of diseases (Leukemia and all kinds of malaria). Image acquisition is based on the collection of 600 images from $U$ and Kaggle sources. Pre-processing takes place in the following stages: preparing the datastore, dividing the data into categories according to above diseases, labeled them to access them for training, verification, and testing.

A sum equal to the number of images for each category to be equivalent to the category with the least number of images. To check the readiness of an image from each category, a $\mathrm{CNN}$ (alexnet resnet50) model was applied as a deep learning application to extract features to detect hematology diagnosis (Leukemia and all kinds of malaria) and classify 
the system's input images. Also, the cross-validation technique was used for training with three folds to reach high accuracy of results.

\section{References}

[1] G. Gunčar et al., "An application of machine learning to haematological diagnosis," Scientific reports, vol. 8, no. 1, pp. 1-12, 2018.

[2] R. Janaki, "Detection of leukemia in microscopic white blood cell images using Gaussian feature convolutional visual recognition algorithm," J Critic Rev, vol. 7, no. 3, pp. 173-180, 2020.

[3] S. H. Kassani, P. H. Kassani, M. J. Wesolowski, K. A. Schneider, and R. Deters, "A hybrid deep learning architecture for leukemic B-lymphoblast classification," in 2019 International Conference on Information and Communication Technology Convergence (ICTC), 2019: IEEE, pp. 271-276.

[4] S. Shafique and S. Tehsin, "Acute lymphoblastic leukemia detection and classification of its subtypes using pretrained deep convolutional neural networks," Technology in cancer research \& treatment, vol. 17, p. 1533033818802789, 2018.

[5] T. Thanh, C. Vununu, S. Atoev, S.-H. Lee, and K.-R. Kwon, "Leukemia blood cell image classification using convolutional neural network," International Journal of Computer Theory and Engineering, vol. 10, no. 2, pp. 54-58, 2018.

[6] S. Rajaraman et al., "Pre-trained convolutional neural networks as feature extractors toward improved malaria parasite detection in thin blood smear images," PeerJ, vol. 6, p. e4568, 2018.

[7] H. A. Mohammed and I. A. M. Abdelrahman, "Detection and classification of malaria in thin blood slide images," in 2017 International Conference on Communication, Control, Computing and Electronics Engineering (ICCCCEE), 2017: IEEE, pp. 1-5.

[8] P. Tiwari et al., "Detection of subtype blood cells using deep learning," Cognitive Systems Research, vol. 52, pp. 1036-1044, 2018.

[9] M. Habibzadeh, M. Jannesari, Z. Rezaei, H. Baharvand, and M. Totonchi, "Automatic white blood cell classification using pre-trained deep learning models: Resnet and inception," in Tenth international conference on machine vision (ICMV 2017), 2018, vol. 10696: International Society for Optics and Photonics, p. 1069612.

[10] A. Rakhlin, A. Shvets, V. Iglovikov, and A. A. Kalinin, "Deep convolutional neural networks for breast cancer histology image analysis," in international conference image analysis and recognition, 2018: Springer, pp. 737-744.

[11] K. Sriporn, C.-F. Tsai, C.-E. Tsai, and P. Wang, "Analyzing Malaria Disease Using Effective Deep Learning Approach," Diagnostics, vol. 10, no. 10, p. 744, 2020.

[12] M. Delgado-Ortet, A. Molina, S. Alférez, J. Rodellar, and A. Merino, "A deep learning approach for segmentation of red blood cell images and malaria detection," Entropy, vol. 22, no. 6, p. 657, 2020.

[13] M. Loey, M. Naman, and H. Zayed, "Deep transfer learning in diagnosing leukemia in blood cells," Computers, vol. 9, no. 2, p. 29, 2020.

[14] A. Bodzas, P. Kodytek, and J. Zidek, "Automated detection of acute lymphoblastic leukemia from microscopic images based on human visual perception," Frontiers in Bioengineering and Biotechnology, vol. 8, p. 1005, 2020.

[15] M. Tahir, T. Badriyah, and I. Syarif, "Classification Algorithms of Maternal Risk Detection For Preeclampsia With Hypertension During Pregnancy Using Particle Swarm Optimization," EMITTER International Journal of Engineering Technology, vol. 6, no. 2, pp. 236-253, 2018.

[16] R. Qu, G. Xu, C. Ding, W. Jia, and M. Sun, "Deep learning-based methodology for recognition of fetal brain standard scan planes in 2D ultrasound images," IEEE Access, vol. 8, pp. 44443-44451, 2019.

[17] P. H. Diniz, "Deep Learning Strategies for Ultrasound in Pregnancy," REPRODUCTIVE HEALTH, 2020. 